Check for updates

Cite this: Phys. Chem. Chem. Phys., $2018,20,8658$

Received 16th October 2017, Accepted 23rd February 2018

DOI: $10.1039 / c 7 c p 07044 f$

rsc.li/pccp

\title{
Remarkable charge-transfer mobility from [6] to [10]phenacene as a high performance $p$-type organic semiconductor $\dagger$
}

\begin{abstract}
Thao P. Nguyen, (D) ${ }^{a}$ P. Roy ${ }^{b}$ and Ji Hoon Shim*ac
The relationship between structure and charge transport properties of phenacene organic semiconductors has been studied with focus on [6] $\rightarrow$ [10]phenacene. Upon inserting phenyl rings, the $\pi$-extended structure results in strong electronic coupling interactions and reduction of reorganization energy. Using the classical Marcus charge transport theory, we predict that hole mobility in the phenacene series increases gradually up to $8.0 \mathrm{~cm}^{2} \mathrm{~V}^{-1} \mathrm{~s}^{-1}$ at [10]phenacene. This is remarkably high among other discovered OSCs, surpassing that of pentacene. Moreover, we notice that the experimental hole mobility of [6]phenacene is unusually low, inconsistent with other members in the same series. Thus, we performed full structural relaxation on phenacene and revealed similarities between theoretical and experimental crystal structures for all the members except [6]phenacene. We propose a new structure of [6]phenacene under the consideration of van der Waals force with smaller lattice parameters $a^{*}$ and $b^{*}$ compared to the experimental structure. Our new structural calculation fits well with the existing trend of hole mobility, energy gaps, effective masses, bandwidth and lattice parameters. Single-shot $G_{0} W_{0}$ calculations are performed to verify our structures. The results give a hint that the improvement in [6]phenacene efficiency lies on the intermolecular distance along the stacking direction of the crystal. Phenacene compounds generally have small effective masses, high charge transfer integrals and moderate reorganization energies necessary for hole transport. Our results suggest that the phenacene series, in particular [6] $\rightarrow$ [10]phenacene, have high charge mobility and air stability essential for achieving high efficiency electronic devices.
\end{abstract}

\section{Introduction}

Organic semiconductors (OSCs) have attracted considerable interest recently as alternative materials for inorganic semiconductors (ISCs) during the last decade. Compared to ISCs, OSCs are flexible and light with advantages of low manufacturing cost, structural tunability and chemical modification. ${ }^{1}$ OSCs have various applications as organic light emitting diodes (OLED), organic field effect transistors (OFETs) and photovoltaic devices. ${ }^{2}$ Pentacene from the acene series has the highest hole mobility observed among all OSCs. ${ }^{3}$ The mobility of pentacene in a thin film transistor reaches about $3 \mathrm{~cm}^{2} \mathrm{~V}^{-1} \mathrm{~s}^{-1}$, surpassing that of amorphous silicon $\left(0.5 \mathrm{~cm}^{2} \mathrm{~V}^{-1} \mathrm{~s}^{-1}\right) .{ }^{4-6}$ In pentacene single crystals, the hole mobility is as high as $35 \mathrm{~cm}^{2} \mathrm{~V}^{-1} \mathrm{~s}^{-1}$, one of the

\footnotetext{
${ }^{a}$ Department of Chemistry, Pohang University of Science and Technology, Pohang 37673, Korea. E-mail: jhshim@postech.ac.kr; Tel: +82-54-279-2344

${ }^{b}$ Theoretical Chemistry, Faculty of Sciences, Radboud University,

Heyendaalseweg 135, 6525 AJ, Nijmegen, The Netherlands

'Division of Advanced Nuclear Engineering, Pohang University of Science and Technology, Pohang 37673, Korea

$\dagger$ Electronic supplementary information (ESI) available. See DOI: 10.1039/c7cp07044f
}

highest reported mobility in OSCs. ${ }^{7}$ Although pentacene carries a small band gap and high charge carrier mobility, the issue in pentacene is its chemical instability during device operation. Pentacene was observed to degrade under an ambient environment leading to dramatic reduction in its charge transport mobility. ${ }^{8}$ Adding more phenyl rings to acene is thought to improve the charge mobility but leads to further degradation, thus leading to device inefficiency. ${ }^{9}$ Until now, pentacene is the biggest member to be synthesized among the acene series and no information about other members larger than six rings has been reported. ${ }^{9}$ Therefore by improving charge mobility and air stability, OSCs are expected to replace silicon in electronic devices. The development of OSCs with both higher charge mobility and air stability still remains a challenge.

Recently, the phenacene series (Fig. 1) have been studied as promising semiconducting materials with significant improvement in air stability due to a deeper HOMO level compared to acene. ${ }^{10}$ Picene as a derivative of pentacene shows a high hole mobility $\left(\mu=1.4-3.2 \mathrm{~cm}^{2} \mathrm{~V}^{-1} \mathrm{~s}^{-1}\right)$, comparable to that of amorphous silicon. ${ }^{11,12}$ It exhibits an increase in hole mobility upon contacting with oxygen in the environment. ${ }^{11}$ Due to the 


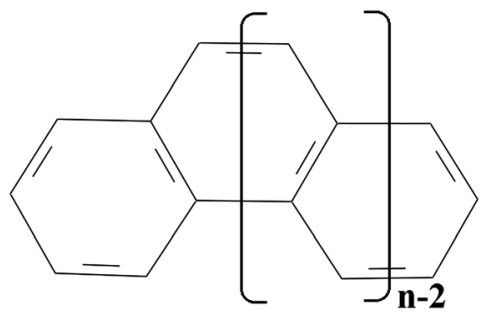

Fig. 1 Chemical structural formula of $[n]$ phenacene ${ }^{17}$ with $n$ being the number of phenyl rings.

stability of the phenacene series in an ambient atmosphere, the members with more than five rings have been successfully fabricated; namely [6]phenacene, [7]phenacene, [8]phenacene and [9]phenacene. A thin film based on [6]phenacene reaches a mobility of $7.4 \mathrm{~cm}^{2} \mathrm{~V}^{-1} \mathrm{~s}^{-1}$ and thin film single-crystal FETs of [7]phenacene and [8]phenacene achieved hole mobilities of 6.9 and $16.4 \mathrm{~cm}^{2} \mathrm{~V}^{-1} \mathrm{~s}^{-1}$, respectively. ${ }^{13-15}$ Recently, [9]phenacene has been synthesized with hole mobility reaching as high as $18 \mathrm{~cm}^{2} \mathrm{~V}^{-1} \mathrm{~s}^{-1}$, which is the highest field effect mobility in OFETs. ${ }^{16}$ Theoretical investigations on these high performance OSCs are needed to reveal the factors responsible for high charge carrier mobility in electronic devices, such as molecular packing of solids, electronic structure, device geometry, conducting angle, interfaces, etc. In this work, we performed a first-principles calculation to predict the charge mobility in the phenacene series and investigate the factors influencing the hopping process in OSC materials.

\section{Computational methods}

The geometry optimizations of the crystal structures are performed using the projector augmented wave (PAW) technique of density functional theory (DFT) with periodic boundary conditions (PBC) as implemented in the Vienna $\mathrm{Ab}$ initio Simulation Package (VASP). ${ }^{18-21}$ For the exchange correlation we use generalized gradient approximation by Perdew, Burke and Ernzerhof (GGA-PBE) ${ }^{22}$ and vdW-DF/revPBE ${ }^{23,24}$ functionals to optimize the crystal structures of [6] $\rightarrow$ [10]phenacene. GGAPBE is a well-known functional to study the properties of strongly bound systems. However, in organic crystals, molecules are bound with each other mainly by a weak van der Waals force in which neither GGA nor LDA is designed. Therefore, vdW-DF/ revPBE has been used in which the van der Waals forces have been taken into account. Next, the electronic band structures are calculated using GGA-PBE levels of theory using the optimized crystal structures. Convergence was achieved with an energy cutoff of $500 \mathrm{eV}$ and a $\Gamma$-centered $k$-point mesh of $4 \times 4 \times 4$ within the irreducible part of the Brillouin zone. Relaxation criteria of $10^{-6} \mathrm{eV}$ for electronic steps and $10^{-5} \mathrm{eV}$ for ionic steps were used.

The $\mathrm{G}_{0} \mathrm{~W}_{0}$ method is employed to obtain the accurate band gaps of the systems. The quasi-particle $(\mathrm{QP}) \mathrm{G}_{0} \mathrm{~W}_{0}$ calculation involves the single-shot correlation to eigenvalues obtained from DFT calculations by consideration of self-energy ( $i \mathrm{GW})$.
Here $\mathrm{G}$ and $\mathrm{W}$ are Green's function and the screening parameter, respectively. ${ }^{25} \mathrm{~W}$ is obtained from the Kohn-Sham wavefunction-based dielectric function. ${ }^{26}$ It is generally calculated by means of linear response theory under random phase approximation (RPA) with local field effects. ${ }^{27}$ One can further improve the QP approximation by means of the self-consistent GW (sc-GW) technique, which includes the effects of screening on the QP wavefunction. ${ }^{28,29}$ However, previous research suggested that the sc-GW technique overestimates the band gap of the system as it neglects the lattice polarization contribution to the screening of the electron-electron interactions. Hence, it is reported that a partial cancellation of the errors leads to a better estimation of the band gap in the $\mathrm{G}_{0} \mathrm{~W}_{0}$ calculation. ${ }^{29}$ Here we performed single shot $\mathrm{G}_{0} \mathrm{~W}_{0}$ calculations using the optimized lattice parameters.

There are two models to calculate the charge transfer process in OSCs: band-like theory and hopping theory. The band theory assumes that the charge carrier electron is delocalized over the crystal, similar to the inorganic semiconductors. The bandwidths of conduction and valence bands determine the hole and electron mobilities, respectively. However, band theory only works well at low temperature. As the temperature increases, the band transport decreases by the phonon-scattering process and charge mobilities reduce. Thus, the charge carrier is localized over single chains and charge transport is governed by the thermally activated hopping mechanism. ${ }^{30}$ Cheng et al. indicates that a simple band model is only applicable for temperatures up to $150 \mathrm{~K}$ and is inapplicable at ambient temperature. ${ }^{31}$ Furthermore, in band theory, only the band diagram of the lattice structure is important and is summarized by effective masses. ${ }^{32-34}$ It works well in inorganic covalently bond materials. However, organic crystals are weakly bound by van der Waals forces and the molecules are more flexible resulting in an off-diagonal electron-phonon coupling. In this case, band theory fails to determine the charge transport properties in OSCs because it neglects the electronphonon coupling which plays an important role in organic crystals. ${ }^{35}$ Thus, band theory should not be used to describe the charge transfer process in phenacenes at ambient temperature where the hopping process dominates. The hopping model assumes that the charge carrier localizes and hops between molecules. It is proved to accurately calculate the charge mobility in several organic systems as it considers the charge transfer integral as well as electron-phonon coupling (reorganization energy)..$^{2,36,37}$

On the basis of Marcus charge transport theory, ${ }^{38-40}$ the performance of OSCs relies on two main factors. The first one is the geometry relaxation of the molecule under the hopping process. During the process, the electrons/holes are exchanged between the neighboring molecules in a crystal. Thus, the energy cost by the internal geometry rearrangement from the neutral to the charged state and vice versa is called the reorganization energy and acts similar to the energy barrier in the charge transport process.

$$
\lambda=\lambda_{1}+\lambda_{2}=\left(E_{4}-E_{1}\right)+\left(E_{2}-E_{3}\right)
$$




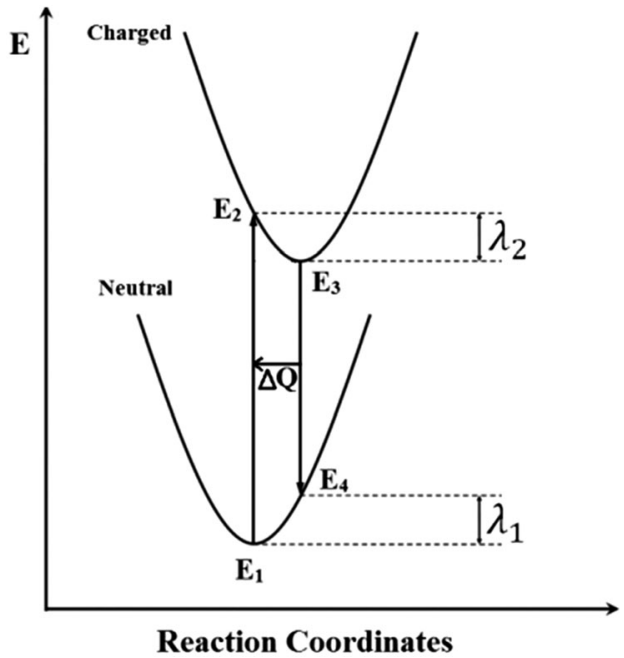

Fig. 2 Schematic description of internal reorganization energy for the hole transfer process.

Here $E_{1}$ is the ground-state energy of a neutral molecule. In Fig. 2, the electrons/holes are hopped from its ground state. As a result, the energy of the neutral molecule changes to a charged state with energy $E_{2}$. At $E_{2}$, the molecule starts to relax its geometry into its ground state energy of the charged state $E_{3}$ which provides the reorganization energy $\lambda_{2}$ in the charged particle. When electrons/holes move out of the charged molecule to the neutral molecule, the energy promptly changes from $E_{3}$ to $E_{4}$, which is the energy of the neutral molecule in the optimized geometry of a charged state. Then, it relaxes its geometry into the ground state energy of the neutral state and provides the reorganization energy $\lambda_{1}$ in the neutral molecule. In the charge transport process between charged and neutral molecules, the sum $\lambda$ of the reorganization energies in each molecule can be considered as an energy barrier.

The second important factor is the magnitude of the electronic coupling or charge transfer integral influenced by the intermolecular interaction of one molecule to its surrounding neighbor molecules. The charge transfer integral is estimated as below:

$$
t_{\mathrm{eff}}=\frac{t_{12}-\frac{1}{2}\left(\varepsilon_{1}+\varepsilon_{2}\right) S_{12}}{1-S_{12}{ }^{2}}
$$

Here, $S_{12}$ is the spatial overlap integral between HOMOs (LUMOs) of two monomers in a dimer, $\varepsilon_{1}$ and $\varepsilon_{2}$ are the site energies of two monomers, and $t_{12}$ is the off-diagonal Fock matrix element between the diabatic molecular orbital basis of the isolated monomer. We note that in the energy splitting in dimer (ESD) method, the charge transfer integral is half of the energy differences between the HOMO and the HOMO-1 (LUMO and LUMO+1) in a dimer. The use of the ESD method usually overestimates the total charge integral since the polarization effect relative to the position of molecules in the crystal space has not been taken into account (eqn (3)). Thus, the effective charge transfer integral can be obtained as eqn (2).
The effective charge transfer integral was calculated using the GGA-PW91 exchange functional theory with the TZP (triple-zeta plus polarization) basis set.

$$
t=\frac{E_{\mathrm{LUMO}+1[\mathrm{HOMO}]}-E_{\mathrm{LUMO}[\mathrm{HOMO}-1]}}{2}
$$

The charge transfer rate $k\left(\mathrm{~s}^{-1}\right)$ is estimated as follows:

$$
k=\frac{4 \pi^{2}}{h} \frac{1}{\sqrt{4 \pi \lambda k_{\mathrm{B}} T}} t_{\mathrm{eff}}^{2} \exp \left(-\frac{\lambda}{4 k_{\mathrm{B}} T}\right)
$$

Here, $k_{\mathrm{B}}$ is the Boltzmann constant, $h$ is Planck's constant and $T$ is the temperature (K). The charge mobility in each hopping pathway is

$$
\mu=\frac{e d^{2} k}{2 k_{\mathrm{B}} T}
$$

where $e$ is the electronic charge and $d$ is the intermolecular distance ( $\mathrm{A})$ between two monomers. The drift mobility of OSCs is evaluated by summing all the possible hopping pathways as

$$
\mu_{\mathrm{drift}}=\frac{e D}{k_{\mathrm{B}} T}
$$

where $D$ is the diffusion coefficient evaluated as $D=\frac{1}{2 n} \sum_{i} d_{i}^{2} k_{i} P_{i}$. The angular anisotropic mobility is defined as ${ }^{41}$

$$
\mu_{\phi}=\frac{e}{2 k_{\mathrm{B}} T} \sum_{i} k_{i} d_{i}^{2} P_{i} \cos ^{2} \gamma_{i} \cos ^{2}\left(\theta_{i}-\phi\right)
$$

where $d_{i}, \gamma_{i}, \theta_{i}$ and $\phi$ depend strongly on the molecular architecture of the organic crystal. In particular, $\gamma_{i}$ is the angle of the hopping jump from one molecule to the plane of its neighboring molecules. In $2 \mathrm{D}$ transport, $\gamma_{i} \approx 0^{\circ} . \theta_{i}$ is the angle of hopping pathways relative to the reference axis, and $\phi$ is the orientation angle of the transistor channel. $P_{i}$ is the probability for charge hopping from one molecule to its $i$ th neighbor molecule: $P_{i}=\frac{k_{i}}{\sum_{i} k_{i}}$.

\section{Results and discussion}

The existing members of phenacenes from [3] to [10]phenacene will be denoted as $3 \mathrm{P} \rightarrow 10 \mathrm{P}$ in this work.

\subsection{Structural relaxation and lattice parameters}

Although the lattice parameters of $6 \mathrm{P} \rightarrow 10 \mathrm{P}$ have been reported in experiments, the atomic positions are not yet known. In order to perform a structural prediction, we construct theoretical structures of $6 \mathrm{P} \rightarrow 10 \mathrm{P}$ based on the crystal structures of $5 \mathrm{P}$ (picene). ${ }^{42} 5 \mathrm{P}$ is a well-known material whose detailed atomic positions and lattice parameters have been carefully investigated in previous reports. ${ }^{43}$ First we add phenyl rings one by one into the $5 \mathrm{P}$ crystal structure. Since these materials are in the same class, we assume that herringbone stacking in the $a b$-plane has been preserved as in $5 \mathrm{P} .{ }^{42}$ In fact, experiments have confirmed the herringbone stacking in the $a b$-plane in $6 \mathrm{P}^{44}$ Then, we optimize the constructed crystal structures using two possible ways. 
In the first set of calculations, we allow the atoms inside the unit cell to be freely relaxed while the lattice parameters are kept constant with experimental values ${ }^{13,16,43,45,46}$ using the restricted PBE-GGA functional (RPG). Constrained unit-cell parameters show very small differences in atomic positions between experimental geometry and optimized geometry. ${ }^{36}$ These structures will be denoted as (3P-RPG $\rightarrow$ 10P-RPG). For the case of $10 \mathrm{P}$, since there is no available experimental report, 10P-RPG was built by following a linear increase in the lattice parameter in accordance with the sizes of $5 \mathrm{P}, 7 \mathrm{P} \rightarrow 9 \mathrm{P}$. In the second set, we relax the whole crystal structure without any constraint in lattice parameters using the vdw-DF/revPBE functional (5P-vdW $\rightarrow$ 10P-vdW) in which the van-der-Waals interaction has been taken into account. The crystal structure parameters have been predicted in Table S1 (ESI $\dagger$ ). As a result, we observe no big differences between the experimental lattice parameters (RPG) and the fully optimized structures using the vdW-DF method (vdW) in $5 \mathrm{P}$ and $7 \mathrm{P} \rightarrow 10 \mathrm{P}$. However, there are significant differences in cell parameters of 6P-RPG and 6P-vdW. The experimental lattice parameter $a^{*}$ is about $12.13 \AA$, which is significantly greater than that obtained in the vdw-DF calculation. Similarly, the experimental lattice parameter $b^{*}$ is $7.9 \AA$, while the vdW-DF method only yields approximately $6.5 \AA$. There is no significant difference in the $c^{*}$ parameter. As previously known, the charge transport of phenacene favors 2D transport in the $a b$ plane, ${ }^{42,47}$ the dramatic difference in $a^{*}$ and $b^{*}$ parameters can alter the charge transport properties of the material.

\subsection{Energy gap}

We calculate the energy gap of single molecule systems using the Gaussian package 09W with the B3LYP/6-31G(d,p) level of theory. ${ }^{51}$ Furthermore, we also calculate the band gap of phenacene crystal structures using PBE-GGA functional theory in both RPG and vdW cases. The experimental gap mentioned here is the optical band gap. Whereas we calculate the electronic band gap using DFT for the ground state of the molecule/lattice. This difference between optical and electronic band gaps is fundamental and these two quantities should not be directly compared. However the trend for the series, as presented in the study (Fig. 3) can be compared. In Fig. 3, the energy gaps of phenacene molecules decrease very slightly upon adding phenyl rings ranging from 3.9 to $4.2 \mathrm{eV}$. In crystal cases, the band gap of vdW structures shows the same decreasing trend as in the case of molecules. However, we see that in RPG cases, the band gap of 6P-RPG does not follow the same inclination but increases making it different from other homologous molecules. Overall, the tendency of the molecular energy gap and crystal structures based vdW indeed agrees well with the experimental optical band gaps.

Since the energy gaps of $6 \mathrm{P}-10 \mathrm{P}$ crystals have not been reported theoretically, we carried out very accurate calculations of band gaps based on $\mathrm{QP} \mathrm{G}_{0} \mathrm{~W}_{0}$ formulation. $\mathrm{G}_{0} \mathrm{~W}_{0}$ is thought to be highly appropriate for the organic systems because the normal GGA-PBE or LDA method tends to underestimate the band gaps in these systems as seen in Fig. 3. Since $G_{0} W_{0}$ is computationally expensive, we only perform the single-shot

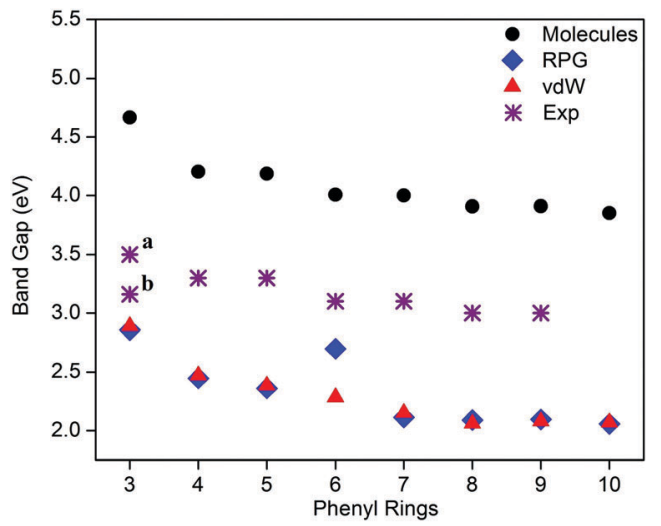

Fig. 3 The calculated energy gaps of both molecules and crystal structures of the phenacene series from 3P to 10P compared to experimental optical energy gaps ${ }^{15,16,48-50}$ available in the literature ( $a$ from ref. 50 and $b$ from ref. 48). For molecules, the B3LYP/6-31G(d,p) level of theory using G09W has been used while GGA-PBE is used for solid state structures.

$\mathrm{G}_{0} \mathrm{~W}_{0}$ calculation for phenacenes from $5 \mathrm{P}$-vdW to $10 \mathrm{P}-\mathrm{vdW}$. We assume that there will be no significant differences in the band gap values of RPG structures and vdW based structures because the lattice parameters of these systems are almost equal to each other. However, due to a dramatic difference in the lattice parameters of 6P-RPG and 6P-vdW, the calculated DOS for both of these systems differ greatly as shown in Fig. 4 .

As seen in Fig. 4, we obtain the density of states (DOS) of the phenacene members. We notice that normally $\mathrm{G}_{0} \mathrm{~W}_{0}$ gives a good theoretical band gap value comparable to the

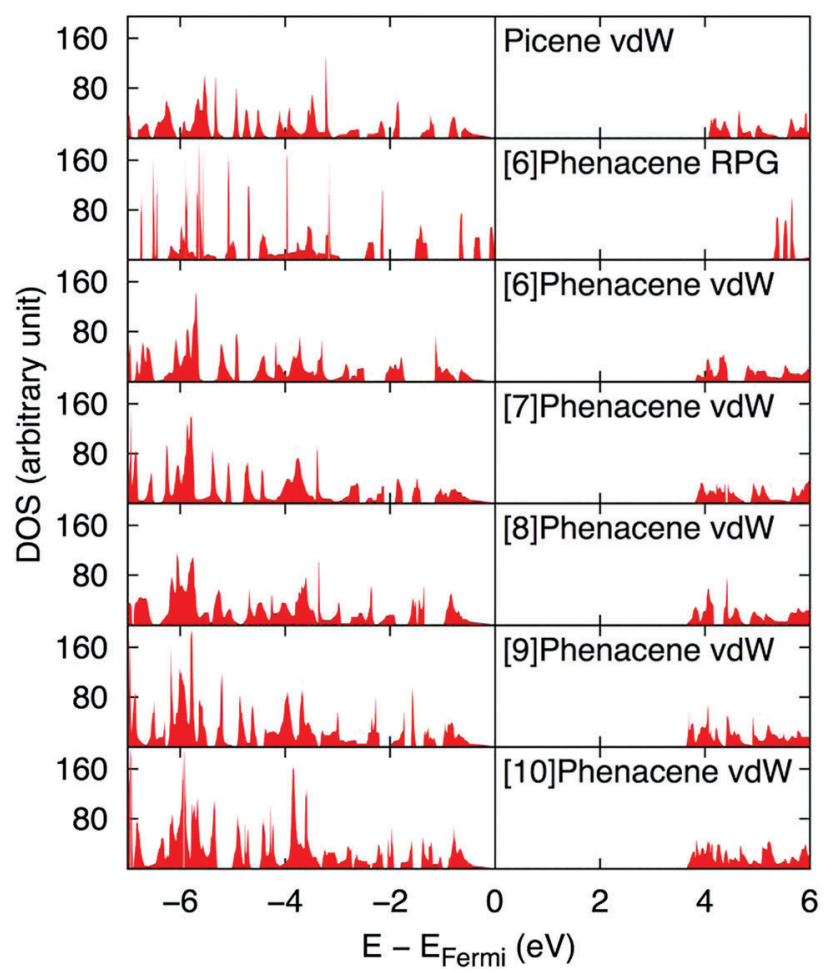

Fig. 4 The density of states of phenacene series from $6 \mathrm{P}-10 \mathrm{P}$ using $\mathrm{G}_{0} \mathrm{~W}_{\mathrm{O}}$ functional theory. 
experimental results. However, in the case of $5 \mathrm{P}$, the $\mathrm{G}_{0} \mathrm{~W}_{0}$ band gap is around $4.0 \mathrm{eV}$, which is larger than the experimental optical band gap of $3.3 \mathrm{eV}$. This overestimation is due to the absence of the excitonic effect in the $\mathrm{G}_{0} \mathrm{~W}_{0}$ calculation. The Bethe-Salpeter equation (BSE) can be used in order to include the electron-hole interaction. Another alternative way to match the absolute values to the experimental band gap is to use TD-DFT that includes excitonic effects. However, due to the sheer size of the system, we avoided both BSE and TD-DFT calculations here. Our calculations agree very well with other reported $\mathrm{G}_{0} \mathrm{~W}_{0}$ calculations of picene. ${ }^{52}$ Moreover, the band gaps from bulk $6 \mathrm{P}-\mathrm{vdW}$ to bulk $10 \mathrm{P}-\mathrm{vdW}$ agree well with the single molecule calculation, ranging from around $3.8 \mathrm{eV}$ to $4 \mathrm{eV}$. The detailed calculated energy gaps yield similar results to the reported values (Table S2, ESI $\dagger$ ). In our previous work, the phenacene group has large band gaps and adding more phenyl rings in the phenacenes does not change the band gap and ionization potential energy significantly $^{42}$ as shown in Fig. 3.

Note that the band gap of $6 \mathrm{P}-\mathrm{RPG}$ is about $5 \mathrm{eV}$ (Fig. 4), much larger than the others, and does not give a similar trend to that in the molecular calculation. This might be due to the larger lattice parameters in 6P-RPG. As a result, the increasing intermolecular distances between neighboring molecules may decrease band dispersion and thus, increase the band gap. Also, according to our optimized calculation, 6P-RPG is energetically less favorable than $6 \mathrm{P}-\mathrm{vdW}$ of $18.89 \mathrm{eV}$ per unit cell, which indicates that 6P-RPG should be assumed as a less stable structure.

\subsection{Effective masses and charge transfer integrals}

It is well known that the mobility is inversely proportional to the effective mass. As shown in Table 1, the hole effective mass is smaller than the electron effective mass. Thus, phenacene groups are favorable to hole transport. Overall, from 3P to 10P, the phenacene group possesses a large valence bandwidth (ranging from $\sim 0.26 \mathrm{eV}$ to $0.45 \mathrm{eV}$ ), along with small hole effective mass, which is necessary for a sufficient hole transfer process. However, we notice that 6P-RPG is clearly different from others, having the largest hole and electron effective

Table 1 Effective hole $\left(m_{\mathrm{h}}{ }^{\star}\right)$ and electron $\left(m_{\mathrm{e}}{ }^{\star}\right)$ mass as a function of molecular length estimated from point $Z$ to $\Gamma$ and from $\Gamma$ to $T$ where the largest valence band dispersion occurs. (Detailed band structures in Fig. S2, ESI) $m_{0}$ denotes the electron rest mass. $\left(^{*}\right)$ correspond to the values calculated from $X$-ray structures. The band dispersion of the valence band from $3 \mathrm{P}$ to $10 \mathrm{P}$ at the $\Gamma$ point

\begin{tabular}{|c|c|c|c|c|}
\hline \multirow[b]{2}{*}{ No. of rings } & \multirow{2}{*}{$\frac{\text { At point } Z-\Gamma}{m_{\mathrm{h}}{ }^{*} m_{0}}$} & \multicolumn{2}{|c|}{ At point $\Gamma-T$} & \multirow{2}{*}{$\frac{\text { Bandwidth }}{\text { VB }}$} \\
\hline & & $m_{\mathrm{h}}{ }^{*} m_{0}$ & $m_{\mathrm{e}}{ }^{*} m_{0}$ & \\
\hline $3^{*}$ & 2.35 & 2.86 & 4.63 & 0.38 \\
\hline $4^{*}$ & 4.85 & 3.40 & 3.36 & 0.22 \\
\hline $5^{*}$ & 1.30 & 1.57 & 2.91 & 0.36 \\
\hline 6P-RPG & 8.38 & 10.09 & 9.84 & 0.03 \\
\hline $6 \mathrm{P}-\mathrm{vdW}$ & 4.05 & 2.16 & 6.27 & 0.26 \\
\hline 7P-RPG & 1.16 & 1.49 & 8.04 & 0.45 \\
\hline 8P-RPG & 1.42 & 1.65 & 3.38 & 0.42 \\
\hline 9P-RPG & 1.40 & 1.17 & 3.62 & 0.38 \\
\hline 10P-RPG & 1.19 & 1.47 & 4.60 & 0.33 \\
\hline
\end{tabular}

masses at the $\Gamma$ point of 10.09 and $9.84 m_{0}^{*}$ respectively. The big hole/electron effective masses come with a flat, narrow valence bandwidth of $0.03 \mathrm{eV}$. These properties in 6P-RPG predict the smallest charge transfer mobility in the series as the hole and electron transfers are immobile. In contrast, the hole effective mass in 6P-vdW is approximately five times smaller than that of 6P-RPG. As a result, a larger valence bandwidth of $0.26 \mathrm{eV}$ is observed. Because of the differences of 6P-RPG with other members and the significant change in the lattice parameters between 6P-RPG and 6P-vdW, we assume that the reported experimental lattice parameters of $6 \mathrm{P}$ may not be the most stable and accurate crystal structure. Thus, we propose a new theoretical structure of 6P in which van der Waals forces have been considered. In the experimental report, it was argued that the difference in lattice parameters of $6 \mathrm{P}$ might come from the even and odd effects, since $4 \mathrm{P}, 6 \mathrm{P}, 8 \mathrm{P}$ and $10 \mathrm{P}$ are in different symmetry groups $\left(C_{2 \mathrm{~h}}\right)$ compared to $3 \mathrm{P}, 5 \mathrm{P}, 7 \mathrm{P}$, and $9 \mathrm{P}\left(C_{2 \mathrm{v}}\right)$ groups. ${ }^{46}$ However, this may not be the case since according to our calculation, $4 \mathrm{P}, 8 \mathrm{P}$ and $10 \mathrm{P}$ band gaps follow the trend well but $6 \mathrm{P}$ is clearly out of the band gap trend (Fig. 3).

In Fig. 5, we report the charge transfer integral calculated from 6P-RPG (vdW) to 10P-RPG (vdW). The charge transfer integral is dominated in channels 1,2 and 3 of the systems, located in the $a b$-plane (Fig. S1, ESI $\dagger$ ). The charge transports in channel 4 and channel 5 along the $c^{*}$ axis are very small and can be ignored. ${ }^{42}$ Since the charge integral depends on the site energies and the orbital overlaps as shown in eqn (2), it is very sensitive to the crystal packing and orientations. Due to the

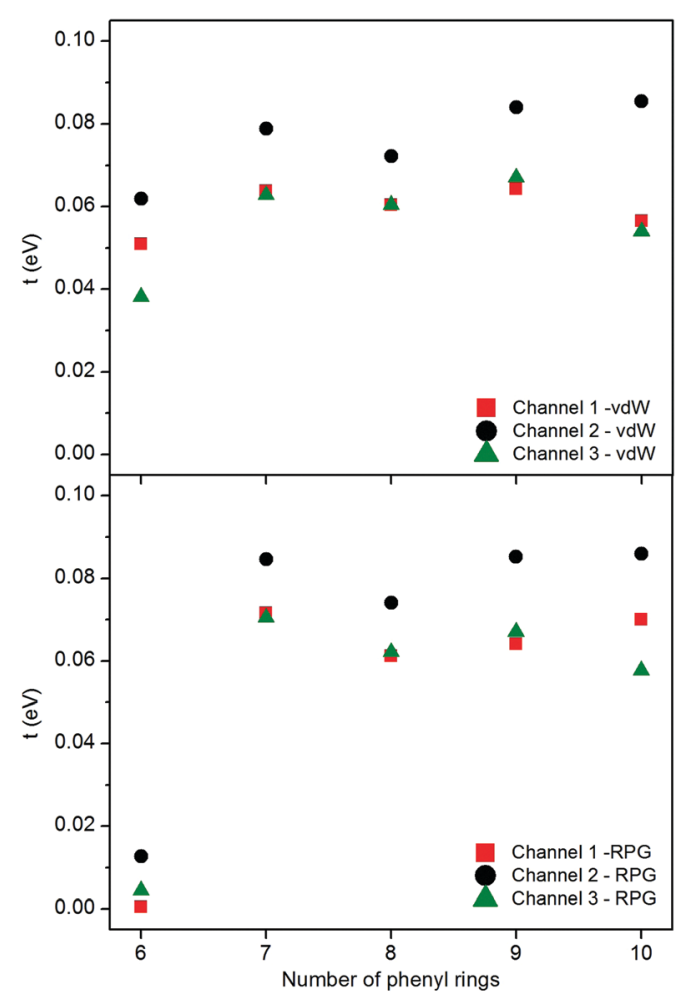

Fig. 5 The hole charge transfer integral of 3P-10P in RPG and vdW cases along channels s1, 2 and 3. 
significant larger lattice parameters in 6P-RPG, the intermolecular distances between monomers in a dimer in 6P-RPG are also longer, giving weaker overlaps of frontier molecular levels (HOMO or LUMO) on different charge localization sites, compared to $6 \mathrm{P}-\mathrm{vdW}$. In addition, because the charge transfer integral depends on the formation of the HOMO (HOMO-1) and the LUMO (LUMO+1) in a dimer, in the concept of bandtransport theory, the charge transfer integral is related to the valence and conduction bandwidths of organic crystals. ${ }^{30}$ The low mobility links to a narrow bandwidth and implies a small charge transfer integral. Also the effective mass is proportional to the curvature of the band. Hence, a narrow band gives heavier effective mass and slow charge transport as a consequence. Thus, the charge transfer integral values in 6P-RPG are predicted to be smaller than those in $6 \mathrm{P}-\mathrm{vdW}$ as seen in Fig. 5. This might be the reason why the experimental data have reported an unusual low mobility in the 6P-RPG material. ${ }^{46}$ In Fig. 5 , channel 2 represents a $\pi-\pi$ stacking dominating the hole transfer integral in the phenacene series and is the most effective charge transfer pathway. Channels 1 and 3 show the herringbone stacking with a lower charge transfer integral. The distances between dimers in channels 4 and 5 increase with the size of the molecules, the hole transfer integrals in these channels are very small and have a small effect on the total hole mobility. The narrow valence band width $(0.03 \mathrm{eV})$ in 6P-RPG, together with heavier hole effective mass, results in the smallest charge transfer integral in the series. Thus, this predicts a very low hole mobility in this material. We note that the use of hybrid functionals like B3LYP which mix Hatree-Fock exchange with the non-local DFT exchange correlation functional can be more suitable to study the properties of the occupied and virtual molecular orbitals with high accuracy on an absolute scale. Thus, the use of hybrid functionals can improve the accuracy of the results. However, it is much more demanding computationally due to the calculation of the Hatree-Fock exchange. Non-hybrid functionals like the GGA-PW91 functional are faster by a factor of 2 and for more localized charge distributions, this can be factors of $3 .^{53}$ Calculations using PW91 are widely used because of its efficiency as well as its comparable results to those determined using B3LYP and BLYP potentials. ${ }^{54}$

\subsection{Reorganization energy and hole mobility}

Besides the charge transfer integral, reorganization energy is also an important parameter that needs to be considered (Fig. 6). Since the charge transfer integral refers to the intermolecular charge transport from one molecule to its neighbors, intramolecular reorganization energies refer to the energy cost when the molecule goes under structural relaxation upon charge hopping. The reorganization energies are determined using the Gaussian 09W package and B3LYP/6-31++G(d,p) from 3P to 8P. Although we are unable to obtain the reorganization energy of 9P and 10P using the same basis sets due to the computational capacity, we perform the calculation using B3LYP/6-31G and B3LYP/6-31G(d,p) for all the phenacene members and obtain the same results with $\mathrm{B} 3 \mathrm{LYP} / 6-31++\mathrm{G}(\mathrm{d}, \mathrm{p})$. Thus, we expect that

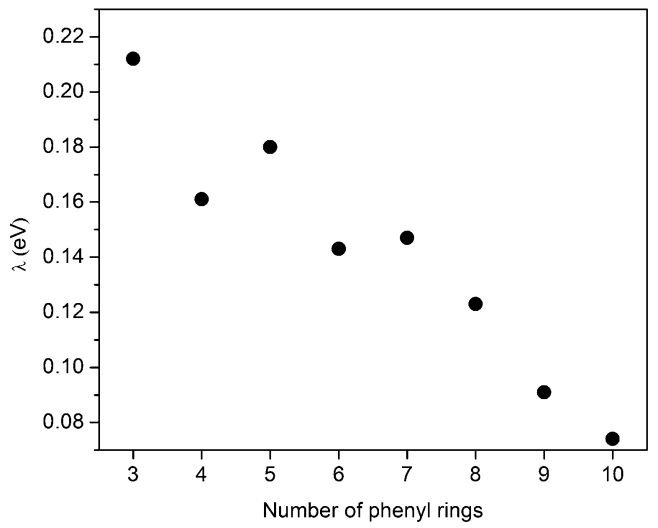

Fig. 6 The reorganization energy of phenacene members at $6-31 G++(d, p)$ from $3 P$ to $8 P$. B3LYP/6-31G and B3LYP/6-31G(d,p) have been used to obtain the reorganization energy of $9 \mathrm{P}$ and $10 \mathrm{P}$ which give the same result in both cases. Our results agree well with the reported data for the case of $3 \mathrm{P} \rightarrow 7 \mathrm{P}^{55}$

the same values of 9P and 10P can be achieved using B3LYP/ $6-31 \mathrm{G}++(\mathrm{d}, \mathrm{p})$. At the first glance, the reorganization energy of the phenacene series follows a zig-zag trend from $3 \mathrm{P}$ to $8 \mathrm{P}$, so we assume that this is due to the even-odd effect in which the even phenacene has a different symmetry from the odd one. However, 9P does not follow this trend and starts to decrease instead of increasing like other odd members in the series. We think that this characteristic occurs because upon adding more phenyl rings from 9P, the length of the molecule is long enough for the charge to localize throughout the molecule and eventually the energy cost for structural relaxation reduces. This property is similar to the reorganization energy in a conjugated polymer chain. As the chain length increases, the reorganization energy decreases continuously and in some cases becomes constant at a certain chain length. ${ }^{56}$ We perform the reorganization energy for $10 \mathrm{P}$ and obtain the values of $0.074 \mathrm{eV}$ at the B3LYP/6-31G(d,p) level of theory, which once again confirms our statement above. We have noticed that the reorganization energy decreased from $0.212 \mathrm{eV}$ to $0.074 \mathrm{eV}$ (Fig. 6). Also the charge transfer integral along channels 4 and 5 (Fig. S1, ESI $\dagger$ ) are extremely small $(\approx 0 \mathrm{eV})$ while the charge transfer integrals are in the range of 0.04 to $0.09 \mathrm{eV}$ in channels 1,2 and 3 (Fig. 5). If we consider the whole crystal where the motion of charge is a homogeneous random walk, the average charge transfer integral in all possible hopping pathways is, therefore, much smaller than the intramolecular vibronic coupling. Thus, the hopping regime can be applied in the case from $3 \mathrm{P}$ to $10 \mathrm{P}$. The diffusion coefficient summing all possible hopping pathways and the drift mobility, therefore, are evaluated (eqn (6)).

Under the concept of Marcus theory, we calculate the hole mobility from $3 \mathrm{P}$ to $10 \mathrm{P}$ as seen in Fig. 7. Around $300 \mathrm{~K}$, the experimental values are reported as $\mu_{\exp }(3 \mathrm{P}) \approx 0.48 \mathrm{~cm}^{2} \mathrm{~V}^{-1} \mathrm{~s}^{-1}, 57$ $\mu_{\exp }(5 \mathrm{P} \rightarrow 8 \mathrm{P}) \approx 1.3,5.6 \times 10^{-1}, 6.9$ and $8.2 \mathrm{~cm}^{2} \mathrm{~V}^{-1} \mathrm{~s}^{-1}, 46$ and $\mu_{\exp }(9 \mathrm{P}) \approx 10.5 \mathrm{~cm}^{2} \mathrm{~V}^{-1} \mathrm{~s}^{-1} \cdot{ }^{16}$ The calculated drift mobilities from 3P-RPG to 10P-RPG are 0.26, 0.40, 0.62, 0.06, 1.30, 1.37, 2.85 and $3.25 \mathrm{~cm}^{2} \mathrm{~V}^{-1} \mathrm{~s}^{-1}$, respectively. It is worth noting that with RPG structures, we obtain a similar trend of drift mobility 


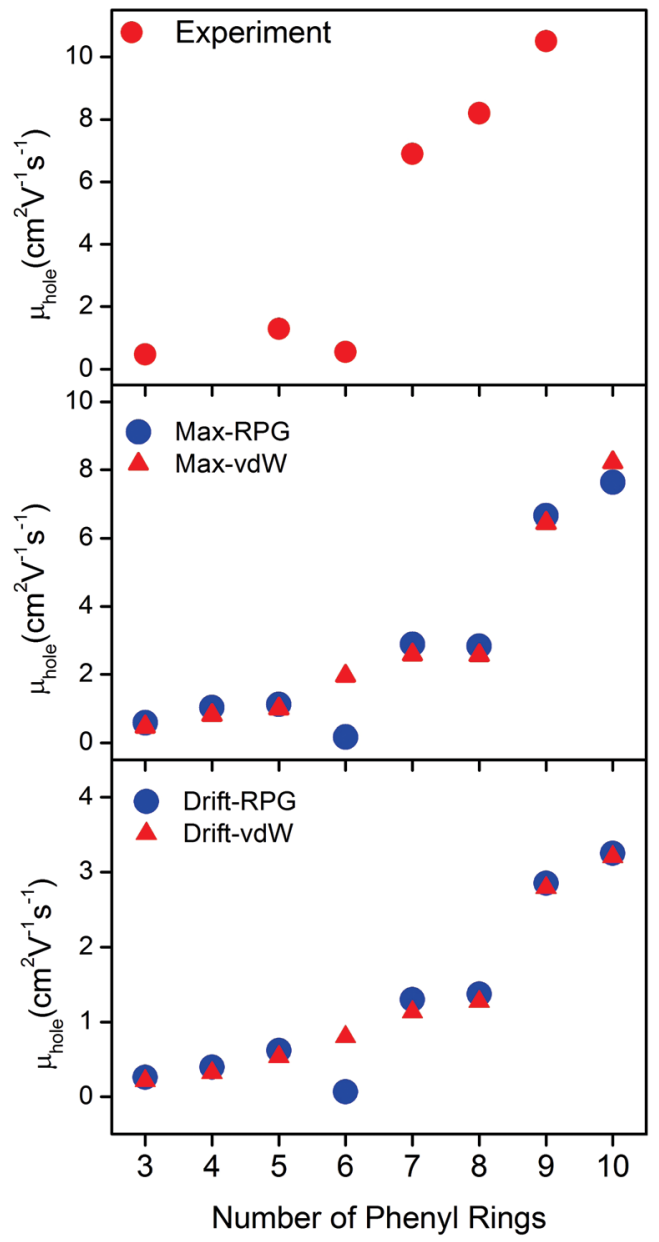

Fig. 7 The hole mobility of 3P-10P in RPG and vdW cases as a function of the molecular length compared to experimental data. ${ }^{16,46,57}$

and maximum mobility between theoretical and experimental structures. Note that the hole mobility of $6 \mathrm{P}$ decreases significantly in contrast to the other phenacene members. In the case of vdW, a similar trend is obtained in $3 \mathrm{P} \rightarrow 5 \mathrm{P}, 7 \mathrm{P} \rightarrow 9 \mathrm{P}$ cases. As mentioned above, our vdW calculation in $6 \mathrm{P}$ gives different structural parameters compared to the experimental one. The lattice of $6 \mathrm{P}-\mathrm{vdW}$ is more stable and more favorable in terms of energy. The smaller intermolecular distances in 6P-vdW grant the material a higher hole mobility than 6P-RPG. The hole mobilities from $3 \mathrm{P}$-vdW to $10 \mathrm{P}-\mathrm{vdW}$ are $0.21,0.32,0.54,0.80$, $1.13,1.27,2.79$ and $3.2 \mathrm{~cm}^{2} \mathrm{~V}^{-1} \mathrm{~s}^{-1}$ respectively. We found that in vdW cases, the hole mobility increases upon inserting phenyl rings which is usual in many conjugated materials. Thus, the odd-even effect is not valid in this case. In fact, a very low mobility in 6P-RPG compared to other members is abnormal. We assume that 6P may produce a better lattice structure with higher hole mobility according to our vdW calculations. Fig. 7 shows a possible exponential trend between hole mobility and the size of $\mathrm{vdW}-[n]$ phenacene. Theoretically, it is possible to find the upper limit of hole mobility when $[n] \rightarrow+\infty$. However our number of data points is insufficient to find the upper limit of the mobility accurately. Hopefully in future studies, calculations with a much longer chain can be conducted to approach this limit.

In summary, vdW-DF gives totally different lattice parameters from the reported experimental ones in $6 \mathrm{P}$ crystals. Besides, the structure of $6 \mathrm{P}-\mathrm{vdW}$ is more stable in terms of energy than 6P-RPG. The energy gap in $6 \mathrm{P}$-vdW is well fitted compared to 6P-RPG which is out of the linear trend. The decrease of the charge transfer integral and hole mobility in 6P-RPG is unusual among the other members of the same group. We suggest that our 6P-vdW structure might be more accurate and can be a promising candidate to improve the low hole mobility of 6P-RPG that has been reported recently. X. He et al. reported that $6 \mathrm{P}$ single crystals in their experiments were not easy to form and the grown crystal size was too small. Therefore, it is believed that the low quality $6 \mathrm{P}$ crystal among phenacene crystals may provide the lowest charge mobility values as well as the formation of the 6P-RPG phase in experiment. ${ }^{45}$

Finally we report the anisotropic mobility from $5 \mathrm{P}$ to $10 \mathrm{P}$ in both RPG and vdW cases (Fig. 8). We can see that in both cases, the phenacene members show similar anisotropic behaviors. The maximum mobility is reached as the conducting transistors create an angle relative to the reference axis of approximately $\phi=90^{\circ}$ or $270^{\circ}$. The minimum mobility is along $\phi=180^{\circ}$ or $360^{\circ}$. The conducting angles are $2.8 \%$ different from our previous report with the X-ray structure of picene which is in the acceptable range. ${ }^{42}$ These small errors arise from the fact that these structures are theoretically constructed, and are relaxed using DFT. The strongest anisotropic transport property is in the $b^{*}$ direction which corresponds to the $\pi-\pi$ stacking transport. This rather agrees with Kubozono et al. that the $\pi-\pi$ interaction increases with the extension of the $\pi$-framework and can be one of the reasons for the increasing hole mobility in phenacene. ${ }^{46}$ However, it is also worth noting that the low reorganization energy has an equally important role in constituting hole mobility. In the RPG case, the mobility of 6P-RPG is the smallest among all members fitting with the reported experimental results. The maximum values of anisotropic mobility from 3P-RPG to 10P-RPG are $0.59,1.02,1.12,0.16$, $2.89,2.84,6.66$ and $7.64 \mathrm{~cm}^{2} \mathrm{~V}^{-1} \mathrm{~s}^{-1}$ respectively. In contrast, vdW-DF shows a gradual increase in the mobility from 3P-vdW to $10 \mathrm{P}-\mathrm{vdW}$. Here we suggested that the highest hole mobility up to approximately $6.4 \mathrm{~cm}^{2} \mathrm{~V}^{-1} \mathrm{~s}^{-1}$ can be obtained in 9P-vdW single crystals and around $8.0 \mathrm{~cm}^{2} \mathrm{~V}^{-1} \mathrm{~s}^{-1}$ in 10P-vdW. We notice that charge hopping rates for much longer chains might be more problematic to evaluate which go beyond the scope of semiclassical Marcus theory. If the reorganization energy reduces further upon adding phenyl rings, the value of the charge transfer integral can, therefore, dominate the whole charge transfer process. As the material becomes perfectly ordered, it is appropriate to use band theory. However, band theory does not provide charge mobility at room temperature. It is not clear which models are better for much longer chains and calculating the "crossover" region where vibronic coupling (reorganization energies) changing from stronger to weaker compared to electronic coupling (charge transfer integral) presents challenges. ${ }^{36}$ It requires future unified theory that 

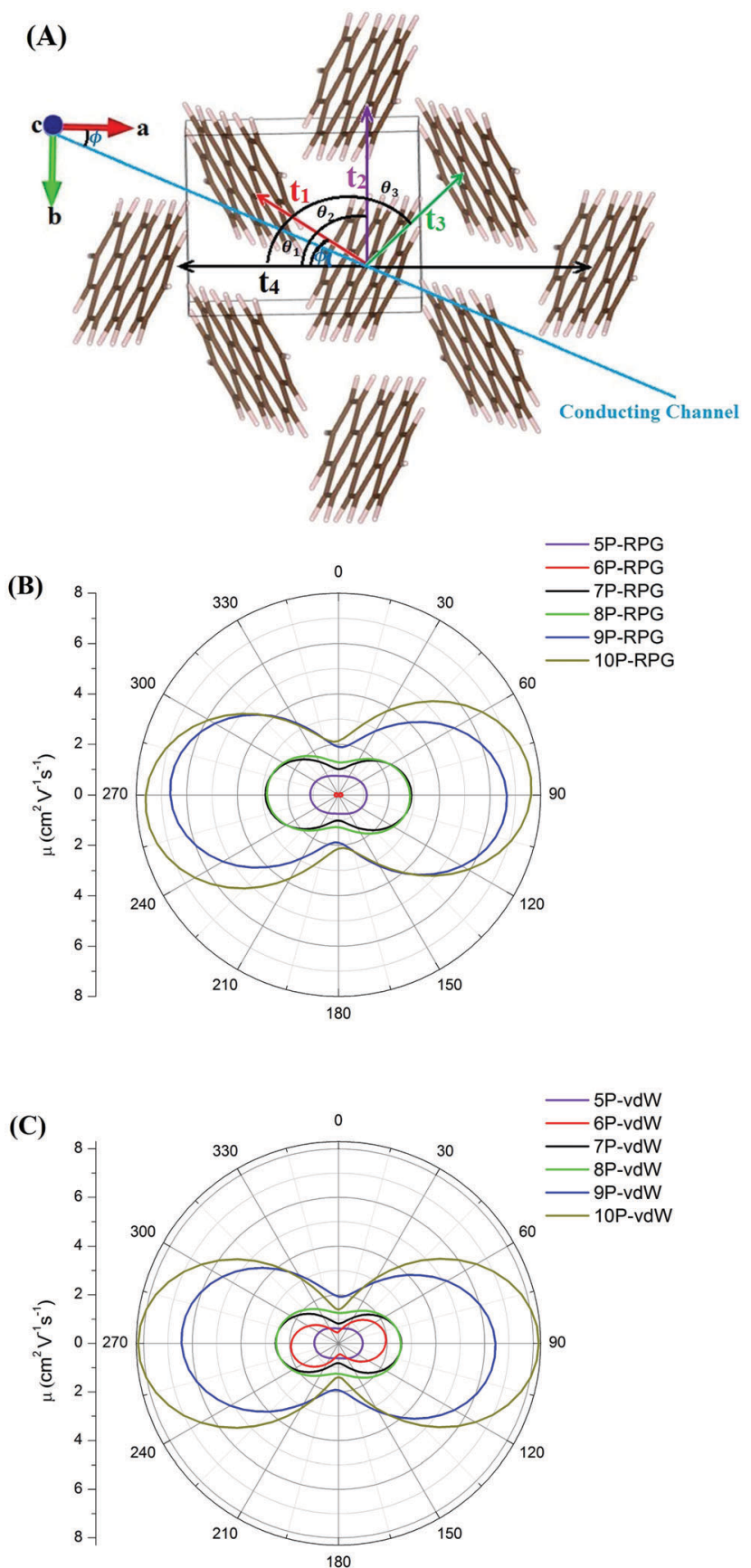

Fig. 8 Illustration of projected hopping pathway schemes in phenacene crystals with respect to the transistor channel in the ab-plane (A). $\theta_{1}, \theta_{2}, \theta_{3}$ and $\theta_{4}$ are the angles along channels $1,2,3$ and 4 relative to the reference crystallographic a axis; $\phi$ is the angle of a transistor channel relative to the reference crystallographic a axis. Polar plots of predicted anisotropic hole mobility along different hopping pathways in the ab plane from 5P to $10 \mathrm{P}$ crystals in both RPG (B) and vaW cases (C). Each circle corresponds to the isovalued mobility depending on its radius as shown in the axis next to the plot.

can fulfill the correct prediction of organic semiconductors. Unfortunately, such a method is not yet available. ${ }^{58}$

Here, it is necessary to mention the limitation of our theoretical method. First, it is true that most of the theoretical calculations so far are based on the experimentally obtained structures.
The dependence of theoretical prediction on the experimental structures has shown the limitation in predicting the properties of new molecules in cases where the knowledge of their crystal structures is unknown. Although the prediction of new crystal structure packing is necessary for future materials research, it remains a critical challenge to many research groups and the current understanding is still limited. In this article, the crystal structures from 6P to 10P are based on the structure of 5P (picene) and the known lattice parameters using the dispersion corrected DFT methods. Due to the lack of experimental crystal database on these materials, the errors in structural prediction are unavoidable with respect to the local and global optimization. However, due to the significant agreement between theoretical and experimental lattice parameters and energy gaps as well as the similarity in the shape of DOS, we believe that our results based on theoretical crystal structures are reasonable enough to give a clear view of the hopping mechanism of phenacenes and suggest a trend of increasing mobility from $3 \mathrm{P}$ to $10 \mathrm{P}$ which have potential application in future advanced electronic devices. Our predicted structures can be used as a reference for the experimental groups for identifying the detailed atomic positions of phenacene crystals. Second, for simplicity, we have used Marcus theory to describe the charge transfer mechanism in phenacene. The application of Marcus theory indeed neglects the dynamic disorder from the thermal fluctuation of the charge transfer integral at high temperature. In the hopping regime of Marcus theory, the intermolecular electronic coupling is kept fixed. However, at room temperature, because the natural bond in organic materials is a weak van der Waals interaction, it is obvious that the orientation of the molecule will fluctuate continuously as the charge transfer integral is modulated by nuclear motions. Therefore, there are several approaches to include the dynamic disorder inside the hopping picture in which the charge transfer integral of the equilibrated system will be calculated after each hopping step at a certain temperature. A phonon-assisted term needs to be added to the temperature dependence of mobility. ${ }^{59}$ However, such type of calculation is computationally expensive in the study of $3 \mathrm{P}-10 \mathrm{P}$ or even larger phenacene structures. It is suggested that in an extreme case, when the fluctuation in the transfer integral is larger than its average, the dynamic disorder can even increase the hole mobility for $2 \mathrm{D}$ materials within the $a b$-plane. ${ }^{59}$ Nevertheless, despite of its limitation, Marcus theory has been widely used to describe the charge transfer mechanism in various organic materials at room temperature and shows good agreement with the experiments. ${ }^{36,38,60}$ The inclusion of dynamic disorder will be considered in our future work for further improvement of the calculated mobility in organic semiconductors. Yet we believe that the increasing trend of mobility in the phenacene series remains unchanged with or without the addition of dynamic disorder.

\section{Conclusion}

We have performed the structural prediction of the phenacene homologous series from $6 \mathrm{P}$ to $10 \mathrm{P}$ using restricted relaxation 
(RPG) as well as a fully geometry relaxation using van der Waals interaction (vdW-DF/revPBE). The energy gaps of phenacene in $\mathrm{vdW}$-DF decrease slightly, agreeing well with the energy gaps of molecules in the gas phase and experimental optical energy gaps. In the RPG case, the energy gap shows the same trend except 6P-RPG. There is a significant difference in the crystal structures between 6P-RPG and 6P-vdW. We calculate the hole mobility from $3 \mathrm{P}$ to $10 \mathrm{P}$ in both RPG and vdW cases. Our hole mobility in the RPG case agrees very well with the reported experimental data with an abnormal small mobility in 6P-RPG. We propose a new crystal structure for $6 \mathrm{P}$ with regard to the vdW interaction that achieves a higher hole mobility than the reported one. We also predict the maximum hole mobility of $10 \mathrm{P}-\mathrm{vdW}$ of around $8.2 \mathrm{~cm}^{2} \mathrm{~V}^{-1} \mathrm{~s}^{-1}$ and the drift mobility of $10 \mathrm{P}$ is approximately $3.2 \mathrm{~cm}^{2} \mathrm{~V}^{-1} \mathrm{~s}^{-1}$. This is the highest among the phenacene series that has been discovered and surpasses the hole mobility of pentacene $\left(3 \mathrm{~cm}^{2} \mathrm{~V}^{-1} \mathrm{~s}^{-1}\right)$. Since the hole mobility will increase as the size of the molecules increase, the synthesis of larger phenacene molecules is necessary. The high mobility might come from dense packing in the crystal. The anisotropy mobility has been proposed in all members of phenacene. The maximum mobility can be achieved when the angles of conducting channels are around $90^{\circ}$ to $270^{\circ}$. The materials show highly anisotropic properties with the $\pi-\pi$ interaction, along with low reorganization energies contributing the highest hole mobility in the $a b$ plane followed by herringbone stacking. We conclude a remarkably high mobility in the phenacene series. Our calculations provide a detailed view of the transport mechanism of OSCs which is not only applicable to phenacene but can also be extended to other organic crystals in the search for good air stability and high mobility OSCs.

\section{Conflicts of interest}

There are no conflicts to declare.

\section{Acknowledgements}

This research was supported by the Global Frontier Program through the Global Frontier Hybrid Interface Materials (GFHIM) of the National Research Foundation of Korea (NRF) funded by the Ministry of Science, ICT \& Future Planning (2013M3A6B1078870).

\section{References}

1 L. Zhu, Y. Yi, Y. Li, E.-G. Kim, V. Coropceanu and J.-L. Brédas, J. Am. Chem. Soc., 2012, 134, 2340-2347.

2 V. Stehr, J. Pfister, R. F. Fink, B. Engels and C. Deibel, Phys. Rev. B: Condens. Matter Mater. Phys., 2011, 83, 155208.

3 Y. Yamashita, Sci. Technol. Adv. Mater., 2009, 10, 024313.

4 T. W. Kelley, D. V. Muyres, P. F. Baude, T. P. Smith and T. D. Jones, Mater. Res. Soc. Symp. Proc., 2003, 771, L6.5.1.

5 W. Wong and A. Salleo, Flexible Electronics: Materials and Applications, Springer, US, 2009.
6 D. Gamota, Printed Organic and Molecular Electronics, Springer, US, 2004.

7 O. D. Jurchescu, J. Baas and T. T. M. Palstra, Appl. Phys. Lett., 2004, 84, 3061-3063.

8 W. L. Kalb, K. Mattenberger and B. Batlogg, Phys. Rev. B: Condens. Matter Mater. Phys., 2008, 78, 035334.

9 R. Mondal, C. Tönshoff, D. Khon, D. C. Neckers and H. F. Bettinger, J. Am. Chem. Soc., 2009, 131, 14281-14289.

10 H. Okamoto, N. Kawasaki, Y. Kaji, Y. Kubozono, A. Fujiwara and M. Yamaji, J. Am. Chem. Soc., 2008, 130, 10470-10471.

11 N. Kawasaki, Y. Kubozono, H. Okamoto, A. Fujiwara and M. Yamaji, Appl. Phys. Lett., 2009, 94, 043310.

12 Y. Kubozono, X. He, S. Hamao, K. Teranishi, H. Goto, R. Eguchi, T. Kambe, S. Gohda and Y. Nishihara, Eur. J. Inorg. Chem., 2014, 3806-3819.

13 R. Eguchi, X. He, S. Hamao, H. Goto, H. Okamoto, S. Gohda, K. Sato and Y. Kubozono, Phys. Chem. Chem. Phys., 2013, 15, 20611-20617.

14 X. He, S. Hamao, R. Eguchi, H. Goto, Y. Yoshida, G. Saito and Y. Kubozono, J. Phys. Chem. C, 2014, 118, 5284-5293.

15 H. Okamoto, R. Eguchi, S. Hamao, H. Goto, K. Gotoh, Y. Sakai, M. Izumi, Y. Takaguchi, S. Gohda and Y. Kubozono, Sci. Rep., 2014, 4, 5330.

16 Y. Shimo, T. Mikami, S. Hamao, H. Goto, H. Okamoto, R. Eguchi, S. Gohda, Y. Hayashi and Y. Kubozono, Sci. Rep., 2016, 6, 21008.

17 H. Okamoto, M. Yamaji, S. Gohda, K. Sato, H. Sugino and K. Satake, Res. Chem. Intermed., 2013, 39, 147-159.

18 G. Kresse and J. Hafner, Phys. Rev. B: Condens. Matter Mater. Phys., 1994, 49, 14251-14269.

19 G. Kresse and J. Hafner, Phys. Rev. B: Condens. Matter Mater. Phys., 1993, 47, 558-561.

20 G. Kresse and J. Furthmüller, Comput. Mater. Sci., 1996, 6, 15-50.

21 G. Kresse and J. Furthmüller, Phys. Rev. B: Condens. Matter Mater. Phys., 1996, 54, 11169-11186.

22 J. P. Perdew, K. Burke and M. Ernzerhof, Phys. Rev. Lett., 1996, 77, 3865-3868.

23 J. Klimeš, D. R. Bowler and A. Michaelides, J. Phys.: Condens. Matter, 2010, 22, 022201.

24 J. Klimeš, D. R. Bowler and A. Michaelides, Phys. Rev. B: Condens. Matter Mater. Phys., 2011, 83, 195131.

25 L. Hedin, Phys. Rev., 1965, 139, A796-A823.

26 G. Onida, L. Reining and A. Rubio, Rev. Mod. Phys., 2002, 74, 601-659.

27 M. Shishkin and G. Kresse, Phys. Rev. B: Condens. Matter Mater. Phys., 2006, 74, 035101.

28 M. van Schilfgaarde, T. Kotani and S. Faleev, Phys. Rev. Lett., 2006, 96, 226402(1).

29 M. Shishkin, M. Marsman and G. Kresse, Phys. Rev. Lett., 2007, 99, 246403.

30 J. L. Brédas, J. P. Calbert, D. A. da Silva Filho and J. Cornil, Proc. Natl. Acad. Sci. U. S. A., 2002, 99, 5804-5809.

31 Y. C. Cheng, R. J. Silbey, D. A. da Silva Filho, J. P. Calbert, J. Cornil and J. L. Brédas, J. Chem. Phys., 2003, 118, 3764-3774. 
32 P. Stallinga, Adv. Mater., 2011, 23, 3356-3362.

33 R. S. Sánchez-Carrera, S. Atahan, J. Schrier and A. AspuruGuzik, J. Phys. Chem. C, 2010, 114, 2334-2340.

34 A. Troisi, Chem. Soc. Rev., 2011, 40, 2347-2358.

35 D. L. Cheung and A. Troisi, Phys. Chem. Chem. Phys., 2008, 10, 5941-5952.

36 V. Coropceanu, J. Cornil, D. A. da Silva Filho, Y. Olivier, R. Silbey and J.-L. Brédas, Chem. Rev., 2007, 107, 926-952.

37 J.-L. Brédas, D. Beljonne, V. Coropceanu and J. Cornil, Chem. Rev., 2004, 104, 4971-5004.

38 M. Huipeng, L. Na and H. Jin-Dou, Sci. Rep., 2017, 1-12.

39 P. F. Barbara, T. J. Meyer and M. A. Ratner, J. Phys. Chem., 1996, 100, 13148-13168.

40 J. Cornil, J.-L. Brédas, J. Zaumseil and H. Sirringhaus, Adv. Mater., 2007, 19, 1791-1799.

41 S.-H. Wen, A. Li, J. Song, W.-Q. Deng, K.-L. Han and I. W. A. Goddard, J. Phys. Chem. B, 2009, 113, 8813-8819.

42 T. P. Nguyen, J. H. Shim and J. Y. Lee, J. Phys. Chem. C, 2015, 119, 11301-11310.

43 A. De, R. Ghosh, S. Roychowdhury and P. Roychowdhury, Acta Crystallogr., Sect. C: Cryst. Struct. Commun., 1985, 41, 907-909.

44 N. Komura, H. Goto, X. He, H. Mitamura, R. Eguchi, Y. Kaji, H. Okamoto, Y. Sugawara, S. Gohda, K. Sato and Y. Kubozono, Appl. Phys. Lett., 2012, 101, 083301.

45 X. He, R. Eguchi, H. Goto, E. Uesugi, S. Hamao, Y. Takabayashi and Y. Kubozono, Org. Electron., 2013, 14, 1673-1682.

46 Y. Shimo, T. Mikami, H. T. Murakami, S. Hamao, H. Goto, H. Okamoto, S. Gohda, K. Sato, A. Cassinese, Y. Hayashi and Y. Kubozono, J. Mater. Chem. C, 2015, 3, 7370-7378.

47 T. P. Nguyen and J. H. Shim, Phys. Chem. Chem. Phys., 2016, 18, 13888-13896.

48 M. T. Bhatti, A. M. Rana, M. Ali, G. N. Shahid and M. Saleh, Turk. J. Phys., 2000, 24, 673-679.

49 F. Roth, B. Mahns, R. Schönfelder, S. Hampel, M. Nohr, B. Büchner and M. Knupfer, J. Chem. Phys., 2012, 137, 114508(1).

50 M. Shoichi, Bull. Chem. Soc. Jpn., 1967, 40, 2749-2753.
51 M. J. Frisch, G. W. Trucks, H. B. Schlegel, G. E. Scuseria, M. A. Robb, J. R. Cheeseman, G. Scalmani, V. Barone, B. Mennucci, G. A. Petersson, H. Nakatsuji, M. Caricato, X. Li, H. P. Hratchian, A. F. Izmaylov, J. Bloino, G. Zheng, J. L. Sonnenberg, M. Hada, M. Ehara, K. Toyota, R. Fukuda, J. Hasegawa, M. Ishida, T. Nakajima, Y. Honda, O. Kitao, H. Nakai, T. Vreven, J. A. Montgomery, Jr., J. E. Peralta, F. Ogliaro, M. Bearpark, J. J. Heyd, E. Brothers, K. N. Kudin, V. N. Staroverov, R. Kobayashi, J. Normand, K. Raghavachari, A. Rendell, J. C. Burant, S. S. Iyengar, J. Tomasi, M. Cossi, N. Rega, J. M. Millam, M. Klene, J. E. Knox, J. B. Cross, V. Bakken, C. Adamo, J. Jaramillo, R. Gomperts, R. E. Stratmann, O. Yazyev, A. J. Austin, R. Cammi, C. Pomelli, J. W. Ochterski, R. L. Martin, K. Morokuma, V. G. Zakrzewski, G. A. Voth, P. Salvador, J. J. Dannenberg, S. Dapprich, A. D. Daniels, O. Farkas, J. B. Foresman, J. V. Ortiz, J. Cioslowski and D. J. Fox, Gaussian09 Revision A.02, Gaussian Inc., Wallingford CT, 2009.

52 S. Yanagisawa, Y. Morikawa and A. Schindlmayr, Jpn. J. Appl. Phys., 2014, 53, 05FY02.

53 B. Baumeier, J. Kirkpatrick and D. Andrienko, Phys. Chem. Chem. Phys., 2010, 12, 11103-11113.

54 M. M. Mikołajczyk, R. Zaleśny, Ż. Czyżnikowska, P. Toman, J. Leszczynski and W. Bartkowiak, J. Mol. Model., 2011, 17, 2143-2149.

55 G. Malloci, G. Cappellini, G. Mulas and A. Mattoni, Chem. Phys., 2011, 384, 19-27.

56 D. Alberga, A. Perrier, I. Ciofini, G. F. Mangiatordi, G. Lattanzi and C. Adamo, Phys. Chem. Chem. Phys., 2015, 17, 18742-18750.

57 N. Karl, Mol. Cryst. Liq. Cryst., 1989, 171, 157-177.

58 F. Ortmann, F. Bechstedt and K. Hannewald, Phys. Status Solidi B, 2011, 248, 511-525.

59 Z. Shuai, L. Wang and C. Song, Hopping Mechanism, Springer Berlin Heidelberg, Berlin, Heidelberg, 2012, pp. 7-41.

60 W. Deng, L. Sun, J. Huang, S. Chai, S. Wen and K. Han, Nat. Protoc., 2015, 10, 632-642. 